\title{
Estrategia estatal de conservación de las cícadas (Zamiaceae): una propuesta para el estado de Hidalgo, México
}

\author{
Aurelia Vite ${ }^{1}$, María T. Pulido ${ }^{*}$ \& Juan C. Flores-Vázquez ${ }^{2}$ \\ 1. Laboratorio de Etnobiología. Centro de Investigaciones Biológicas. Instituto de Ciencias Básicas e Ingenierías. \\ Universidad Autónoma del Estado de Hidalgo, km 4.5 carretera Pachuca-Tulancingo. Pachuca, Hidalgo, Código \\ Postal 42184, México; vite_bios@yahoo.com.mx,mtpulido@yahoo.com \\ 2. Sociedad para el Estudio de los Recursos Bióticos de Oaxaca (SERBO, A. C), Camino Nacional 80a, San Sebastián \\ Tutla, Oaxaca, Código Postal 71246, México; skatoflores@hotmail.com \\ * Correspondencia
}

Recibido 16-VII-2012. Corregido 06-I-2013. Aceptado 08-II-2013.

\begin{abstract}
State strategy for Cycad (Zamiaceae) conservation: A proposal for the State of Hidalgo, Mexico. Mexico has the second largest cycad diversity in the world, and the Sierra Madre Oriental (SMO) is one of the richest biogeographic regions for these plants. Despite there is a general Cycad National Program in the country, there are no state-level cycad conservation strategies or programs. Thus the aim of this study was to propose a cycad conservation strategy for the state of Hidalgo, which is located in the Southern part of the SMO. For this, a cycad species inventory was made in the state, for which three methods were used: review of published literature; consultation in the main Mexican herbaria to verify botanical specimens; and exhaustive field research to compare findings with previously reported species and to recognize new records at the county and state level. The proposed research work strategy combined the following elements: prioritize the county and local areas with greatest cycad species richness; prioritize the species least resistant to environmental change and/or having restricted geographic distribution; and to consider the main uses of these plants by local residents. The results showed that Hidalgo has three genera and eight species of cycads: Ceratozamia fuscoviridis, C. latifolia, C. mexicana, C. sabatoi, Dioon edule, Zamia fischeri, Z. loddigesii and Z. vazquezii, all of which are considered by the International Union for Conservation of Nature (IUCN). This study added two new species records for Hidalgo and 21 at the county level. The species are distributed in 26 counties, of which Chapulhuacán and Pisaflores are notable for their high species richness. Hidalgo has the fourth-greatest cycad species richness among Mexican states, although its area accounts for only $1.07 \%$ of the country. The state's diversity is greater than in other states with larger area, and even than in some other entire countries in Mesoamerica. The presented state cycad conservation strategy proposes that a total of some 11325 ha to be conserved in nine zones, including different vegetation types, distributed in seven counties. The strategy involves a mixed scheme that incorporates conservation in Protected Natural Areas (ANP), Small Farmer Reserves (Reservas Campesinas) and Environmental Management Units (UMA in Spanish). This proposal will be useful for government agencies to take into account in the process of designating land use for the Cloud Forest Biological Corridor (CBBMM in Spanish), a ANP in creation. The state of Hidalgo urgently needs a detailed analysis of trends in changes in vegetation cover and land use, and demographic studies of the cycads. It is recommended that the implementation phase of this state strategy be carried out jointly with local communities, academia, and state and federal agencies responsible for biodiversity conservation. Rev. Biol. Trop. 61 (3): 1119-1131. Epub 2013 September 01.
\end{abstract}

Key words: cycad, Community-based conservation, Cycad National Program, Sierra Madre Oriental, UMAS.

México es el segundo país con mayor riqueza de cícadas en el mundo, con un registro de más de 50 especies de los géneros Ceratozamia Brongn., Dioon Lindl. y Zamia L. Aproximadamente el 80\% de estas especies son endémicas (Hill, Stevenson \& Osborne 2007, Vovides 2000).

La conservación de las cícadas es una prioridad en muchos países debido a que están amenazadas principalmente por el comercio ilegal 
y el cambio de uso del suelo (INE-SEMARNAP 2000). Por ello, muchas de estas especies están incluidas en la Lista Roja de la Unión Internacional para la Conservación de la Naturaleza (UICN) y en la Convención sobre el Comercio Internacional de Especies Amenazadas de Fauna y Flora Silvestre (CITES). En México 50 especies están incluidas en la NOM059-SEMARNAT-2010 (SEMARNAT 2010); además, se ha formulado el Programa Nacional de Cícadas (PNC), cuyo principal objetivo es la conservación, recuperación, manejo y aprovechamiento sustentable de este recurso (INE-SEMARNAP 2000).

El PNC presenta cinco acciones generales:

1) Plantear medidas de protección por especie.

2) Fomentar las Unidades de Manejo de la Vida Silvestre, las UMAS, instrumentos para la conservación y el aprovechamiento de la vida silvestre, que incorporan explícitamente el trabajo de las comunidades locales para proteger, vigilar y aprovechar sosteniblemente la vida silvestre. 3) Capacitar a las autoridades encargadas de la vigilancia y la protección de la vida silvestre. 4) Difundir la importancia de las cícadas entre el público en general. 5) Incrementar el conocimiento científico de estas plantas (INE-SEMARNAP 2000). Este programa reconoce que uno de los problemas existentes para lograr la conservación es la falta de exploración del territorio nacional y señala que es importante establecer estrategias estatales de conservación (INE-SEMARNAP 2000).

En la República Mexicana la mayor riqueza de cícadas, el $34 \%$, se encuentra en la provincia biogeográfica de la Sierra Madre Oriental (SMO) (Yáñez 2006), que también sobresale por la riqueza y los endemismos de otras plantas (Luna et al. 2000, Juárez, Contreras \& Luna 2009) y animales como los reptiles (Canseco-Márquez, Mendoza-Quijano \& Gutiérrez-Mayén 2004) y escarabajos (Márquez \& Morrone 2004), entre otros (Luna, Morrone \& Espinosa 2004).

A pesar de la clara importancia que representa la SMO para la biodiversidad de México, tan solo entre el 5 y el 10\% de su territorio está incluido dentro de Áreas Naturales Protegidas
(ANP), de acuerdo con el análisis de Mas \& Pérez (2005). Las ANP son zonas del territorio nacional en donde los ambientes originales no han sido alterados por la actividad humana, de acuerdo con la Ley General del Equilibrio Ecológico y la Protección al Ambiente (Congreso de la Unión, 1988). Aunque las ANP son la principal estrategia de conservación que se aplica de manera oficial en México (Sarukhán et al. 2009), estas no siempre coinciden con los sitios en los que se encuentran las especies con algún grado de amenaza, como lo mostró el estudio de Luna et al. (2006), en el que se analizó la distribución de 24 especies amenazadas no limitadas a la SMO (incluyendo cícadas) y se concluyó que muchas están subrepresentadas.

La conservación biológica en la SMO es aún más crítica, debido a que no se conoce suficientemente la composición de especies ni su distribución a escalas geográficas detalladas (v.g. municipios). Su extremo sur, por ejemplo, que corresponde políticamente al territorio del estado de Hidalgo, ha sido relativamente poco estudiado a nivel biológico. Aquí actualmente se está buscando decretar y zonificar una nueva ANP conocida como "Corredor Biológico del Bosque Mesófilo de Montaña" (CBBMM) (A. López Portillo com. pers.).

El presente estudio es una contribución al conocimiento de la diversidad de cícadas de este territorio, que muestra una gran riqueza, y el primer planteamiento de una estrategia para su conservación. Este puede servir como un estudio de caso aplicable a otras zonas con gran riqueza biológica. Los objetivos específicos son documentar la distribución de la riqueza de especies de cícadas a nivel municipal, seleccionar las áreas prioritarias para su conservación y comparar la riqueza de zamiáceas de Hidalgo con otras zonas geográficas.

\section{MATERIALES Y MÉTODOS}

Lugar de estudio: El estado de Hidalgo se ubica en la parte central de México, tiene una superficie de $20905 \mathrm{~km}^{2}$, equivalente al $1.07 \%$ del territorio mexicano y está dividido en 84 
municipios (INEGI, 1992). Su territorio corresponde en su mayor parte a la provincia biogeográfica de la SMO y en menor proporción al Eje Neovolcánico Transversal y la Llanura Costera del Golfo Norte.

La SMO está constituida principalmente por rocas sedimentarias continentales y marinas, entre las que predominan las calizas $\mathrm{y}$ en segundo término las rocas arcillosas y las areniscas. También hay, superpuestos, extensos derrames de rocas volcánicas, tales como los basaltos y tobas. El Eje Neovolcánico Transversal cubre la porción sur del estado y está constituido predominantemente por rocas volcánicas. La Llanura Costera solo cubre una pequeña porción del norte del estado caracterizado por rocas sedimentarias de origen marino (INEGI 1992). Hidalgo posee variadas formaciones vegetales que incluyen desde secos matorrales xerófilos hasta bosques de coníferas y bosque mesófilo de montaña (Rzedowski 2006).

Planteamiento de la estrategia estatal: Las estrategias para la conservación de la biodiversidad claramente requieren de estudios y esfuerzos a diversas escalas geográficas (nacional, regional y local) y con distintos enfoques (temáticos, ecosistémicos, especies de interés, etc.), los cuales deben actualizarse de manera periódica (March et al. 2009). Algunos de los criterios empleados fueron: priorizar los municipios y localidades con riqueza sobresaliente de cícadas, priorizar las especies menos resistentes a cambios en el ambiente o con una distribución geográfica reducida y considerar el grado de presión humana sobre estas plantas.

Revisión de colecciones botánicas y recorridos de campo: Para documentar la riqueza de especies de cícadas a nivel estatal y municipal se revisó la bibliografía existente (Contreras, Luna \& Alcántara, 2001, 2003, Luna \& Alcántara 2004, Yáñez 2006, Mejenes, Barragán \& Hernández 2007, The Cycad Pages 2009); se examinaron los ejemplares botánicos recolectados en el estado de Hidalgo y depositados hasta noviembre 2009 en el Herbario Nacional de México (MEXU), el Herbario del Centro de Investigaciones Biológicas de la Universidad Autónoma del Estado de Hidalgo (HGOM), el Herbario del Instituto de Ecología, A.C. (IEB), el Herbario de la Facultad de Ciencias de la UNAM (FCME) y el Herbario de la Escuela Nacional de Ciencias Biológicas (ENCB). Los ejemplares más antiguos de cícadas recolectadas en Hidalgo fueron de los años 1957 en MEXU, 1964 en ENCB, 1968 en FCME y 1992 en IEB.

Considerando lo anterior, se realizaron recorridos de campo (de diciembre 2007 a junio 2011) y recolectas botánicas en 19 municipios (Atlapexco, Chapulhuacán, Jacala de Ledezma, Juárez Hidalgo, Huautla, Huazalingo, La Misión, Molango de Escamilla, Pacula, Pisaflores, San Bartolo Tutotepec, San Felipe Orizatlán, Tepehuacán de Guerrero, Tianguistengo, Tlahuiltepa, Tlanchinol, Xochicoatlán, Xochiatipan y Zimapán) (Fig. 1). Se recolectaron 53 ejemplares de herbario bajo la numeración del primer autor (AVR) y se depositó el material en los herbarios HGOM y XAL (Herbario del Instituto de Ecología, A.C., México). Los ejemplares fueron identificados con claves taxonómicas (Vovides 1999, Contreras et al. 2001, 2003) y fueron posteriormente determinadas o revisadas por el Dr. Andrew P. Vovides, especialista mexicano en cícadas.

Selección de áreas: Para elegir las áreas con mayor prioridad para la conservación de cícadas se ubicaron las zonas de mayor riqueza. Para comparar la riqueza de especies entre zonas con distinta extensión territorial se empleó el método usado por Squeo et al. (1998), que calcula un índice de biodiversidad (IB) al dividir el número de especies (S) encontradas en un área, entre el logaritmo natural del área $(\ln \mathrm{A})$. Así, $\mathrm{IB}=\mathrm{S} / \ln \mathrm{A}$. Para todo $\mathrm{A}>1$, este índice puede tomar valores entre cero e infinito, siendo mayor la diversidad de un área a mayor valor del índice. El área se calculó en $\mathrm{km}^{2}$. 


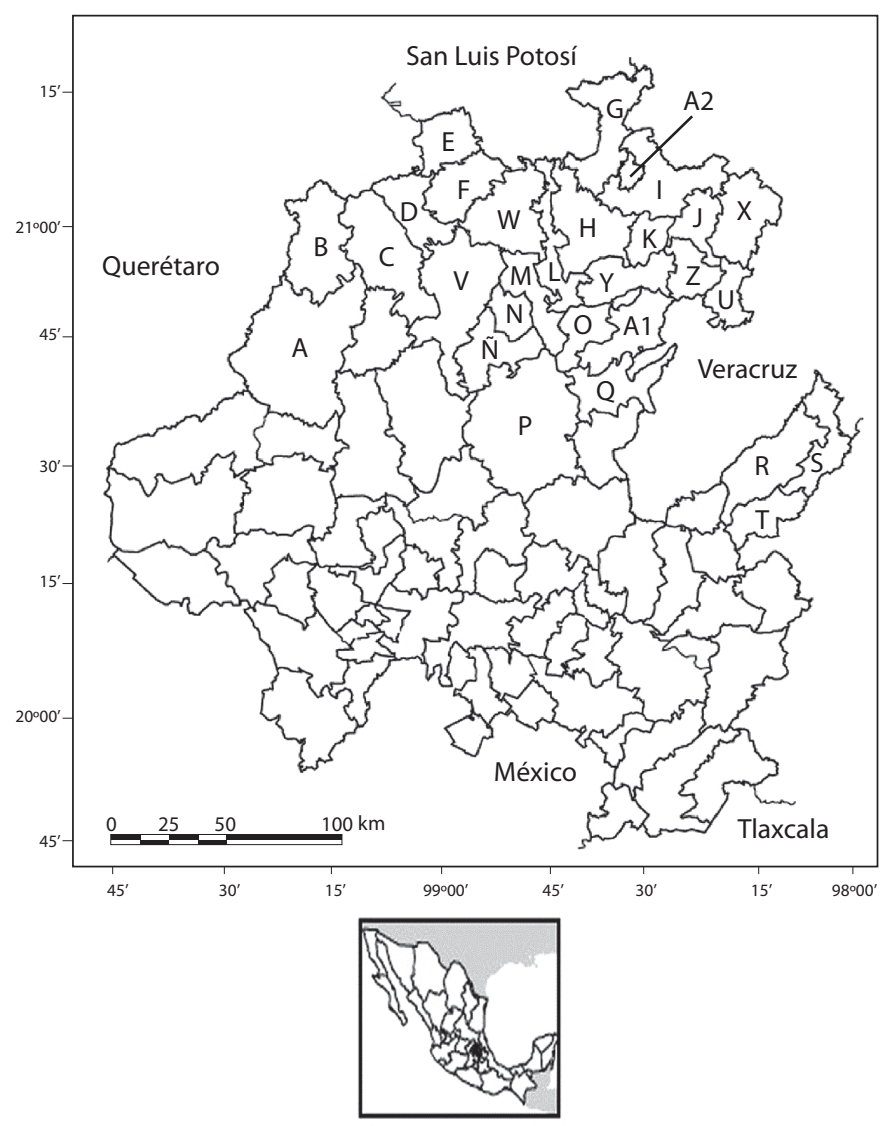

Fig. 1. Municipios del estado de Hidalgo con presencia de cícadas: Zimapán (A), Pacula (B), Jacalade Ledezma (C), La Misión (D), Pisaflores (E), Chapulhuacán (F), San Felipe Orizatlán (G), Tlanchinol (H), Huejutla de Reyes (I), Atlapexco (J), Huazalingo (K), Lolotla (L), Molango de Escamilla (M), Juárez Hidalgo (N), Eloxochitlán (Ñ), Xochicoatlán (O), Metztitlán (P), Zacualtipán de Ángeles (Q), San Bartolo Tutotepec (R), Huehuetla (S), Tenango de Doria (T), Xochiatipan (U), Tlahuiltepa (V), Tepehuacán de Guerrero (W), Huautla (X), Tianguistengo (A1). Municipios que faltan por muestrear: Calnali (Y), Yahualica (Z), Jaltocán (A2).

Fig. 1. Municipalities of Hidalgo State where cycads have been reported: Zimapán (A), Pacula (B), Jacalade Ledezma (C), La Misión (D), Pisaflores (E), Chapulhuacán (F), San Felipe Orizatlán (G), Tlanchinol (H), Huejutla de Reyes (I), Atlapexco (J), Huazalingo (K), Lolotla (L), Molango de Escamilla (M), Juárez Hidalgo $(\mathrm{N})$, Eloxochitlán (Ñ), Xochicoatlán $(\mathrm{O})$, Metztitlán (P), Zacualtipán de Ángeles (Q), San Bartolo Tutotepec (R), Huehuetla (S), Tenango de Doria (T), Xochiatipan (U), Tlahuiltepa (V), Tepehuacán de Guerrero (W), Huautla (X), Tianguistengo (A1). Municipalities that have yet to sample: Calnali (Y), Yahualica (Z), Jaltocán (A2).

\section{RESULTADOS}

\section{Distribución de la riqueza de cícadas en}

Hidalgo: La riqueza del estado incluye un total de ocho especies: Ceratozamia fuscoviridis Moore, Ceratozamia sabatoi Vovides, Vázq. Torres, Schutzman \& Iglesias, Ceratozamia latifolia Miq., Ceratozamia mexicana Brongn.,
Dioon edule Lindl., Zamia fischeri Miq., Zamia loddigesii Miq., y Zamia vazquezii D.W. Stev., Sabato, A. Moretti \& De Luca (Fig. 2).

De acuerdo con la información recabada en herbarios y recorridos de campo, se reconoce que las cícadas se distribuyen al menos en 26 municipios del norte de Hidalgo, destacando Chapulhuacán y Pisaflores, como los 

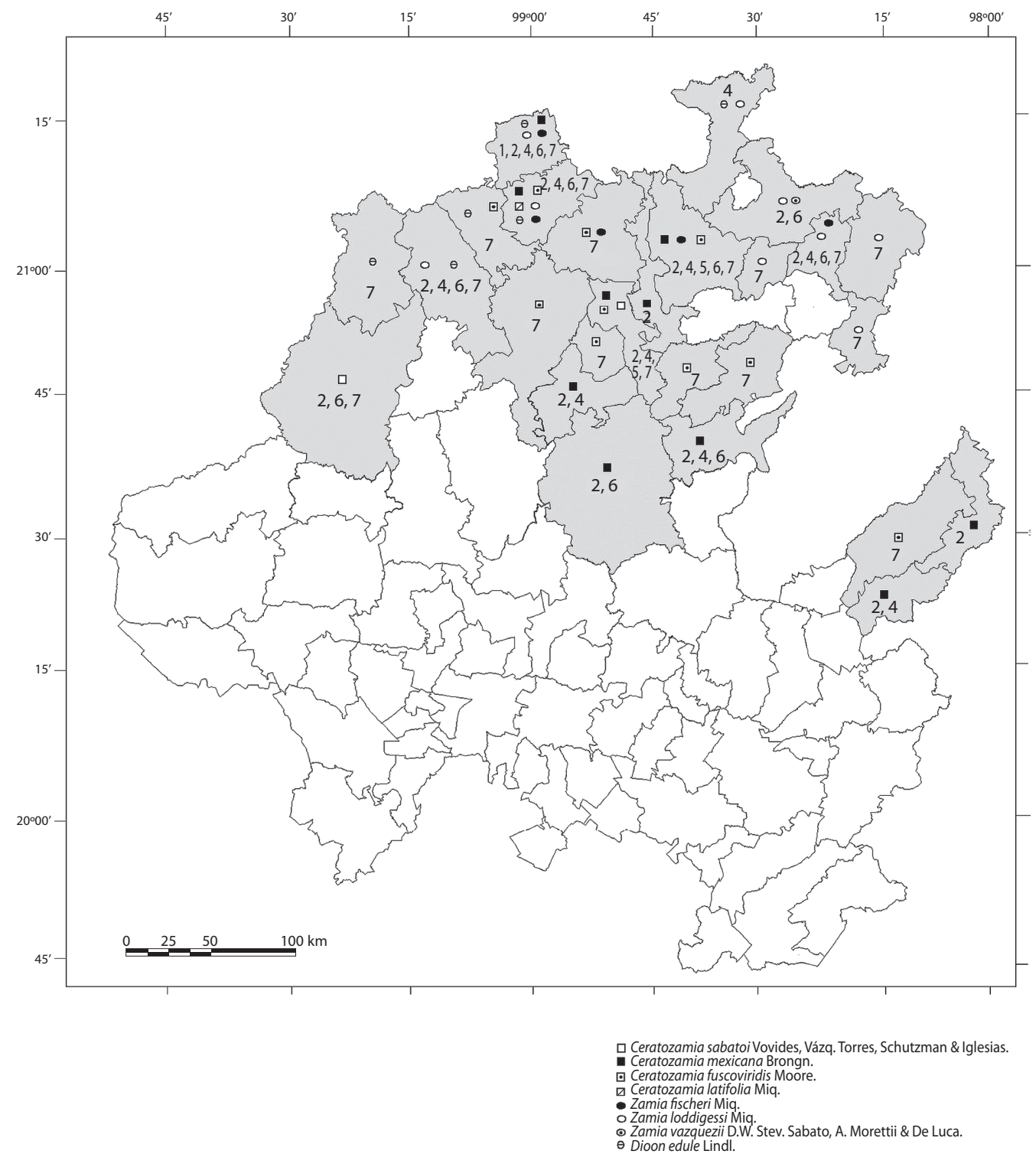

Fig. 2. Distribución de las especies de cícadas presentes en los municipios del estado de Hidalgo. Los números indican la fuente de donde se obtuvo la información: 1. Contreras et al. 2001, 2. Contreras et al. 2003, 3. Yáñez 2006, 4. Mejenes et al. 2007, 5. Osborne et al. 2006, 6. Ejemplares de herbarios (MEXU, IEB, FCME, ENCB, HGOM), 7. Recolectas botánicas (Aurelia Vite Reyes, María T. Pulido y Juan C. Flores-Vázquez).

Fig. 2. Cycad species distribution in municipalities of Hidalgo State. Numbers correspond to consulted references: 1. Contreras et al. 2001, 2. Contreras et al. 2003, 3. Yáñez 2006, 4. Mejenes et al. 2007, 5. Osborne et al. 2006, 6. Herbaria specimens (MEXU, IEB, FCME, ENCB, HGOM), 7. Botanical collections (Aurelia Vite, María T. Pulido y Juan C. Flores-Vázquez). 
de mayor riqueza con seis y cuatro especies (1.10 y 0.79 especies/ln área, respectivamente); mientras que los otros municipios presentan tan solo entre 0.15 a 0.54 especies/ln área (Cuadro 1, Fig. 2).
El estudio permitió dos nuevos registros de especies para Hidalgo y 21 a nivel municipal. Las especies que no habían sido reportadas para el estado son: C. latifolia (datos de campo) y Z. vazquezii (datos de herbario, ejemplar

\section{CUADRO 1}

Especies de cícadas reportadas en la literatura, recolectas depositadas en herbarios y datos de campo, para los municipios del estado de Hidalgo

TABLE 1

Cycad species distributed in the State of Hidalgo, according to literature, herbaria records and fieldwork

\begin{tabular}{|c|c|c|c|c|c|c|c|c|c|c|}
\hline Municipio & 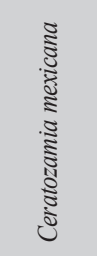 & 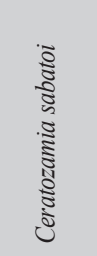 & 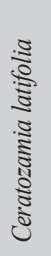 & 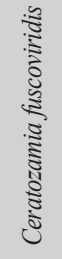 & 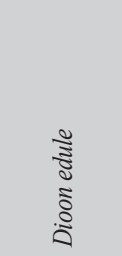 & 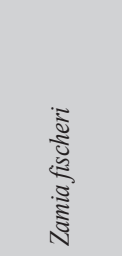 & 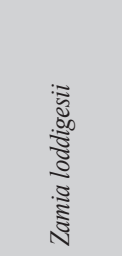 & 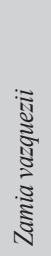 & 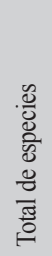 & 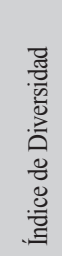 \\
\hline Chapulhuacán & $2,4,6$ & & 7 & 7 & 7 & 7 & 7 & & 6 & 1.10 \\
\hline Pisaflores & 2,4 & & & & 7 & $1,2,4,6$ & $2,4,6,7$ & & 4 & 0.79 \\
\hline Molango de Escamilla & 2,4 & $2^{*}$ & & 5,7 & & & & & 3 & 0.54 \\
\hline Tlanchinol & $2,4,6$ & & & 5,7 & & 7 & & & 3 & 0.50 \\
\hline Atlapexco & & & & & & 7 & $2,4,6$ & & 2 & 0.45 \\
\hline La Misión & & & & 7 & 7 & & & & 2 & 0.39 \\
\hline San Felipe Orizatlán & & & & & 4 & & 4,7 & & 2 & 0.35 \\
\hline Jacala de Ledezma & & & & & $2,4,6,7$ & & 2 & & 2 & 0.34 \\
\hline Huejutla de Reyes & & & & & & & 2,6 & 6 & 2 & 0.34 \\
\hline Tepehuacán de Guerrero & & & & 7 & & 7 & & & 2 & 0.33 \\
\hline Huazalingo & & & & & & & 7 & & 1 & 0.21 \\
\hline Juárez Hidalgo & & & & 7 & & & & & 1 & 0.20 \\
\hline Tianguistengo & & & & 7 & & & & & 1 & 0.20 \\
\hline Xochiatipan & & & & & & & 7 & & 1 & 0.20 \\
\hline Xochicoatlán & & & & 7 & & & & & 1 & 0.20 \\
\hline Eloxochitlán & 2,4 & & & & & & & & 1 & 0.19 \\
\hline Lolotla & 2 & & & & & & & & 1 & 0.19 \\
\hline Tenango de Doria & 2,4 & & & & & & & & 1 & 0.19 \\
\hline Huautla & & & & & & & 7 & & 1 & 0.18 \\
\hline Huehuetla & 2 & & & & & & & & 1 & 0.18 \\
\hline Zacualtipán de Ángeles & $2,4,6$ & & & & & & & & 1 & 0.18 \\
\hline San Bartolo Tutotepec & & & & 7 & & & & & 1 & 0.17 \\
\hline Pacula & & & & & 7 & & & & 1 & 0.16 \\
\hline Tlahuiltepa & & & & 7 & & & & & 1 & 0.16 \\
\hline Metztitlán & 2,6 & & & & & & & & 1 & 0.15 \\
\hline Zimapán & & $2,6,7$ & & & & & & & 1 & 0.15 \\
\hline No especificado & 3 & & 3 & & 3 & & 3 & & & \\
\hline Total & 10 & 2 & 1 & 10 & 6 & 5 & 9 & 1 & & \\
\hline
\end{tabular}

Los números representan la fuente bibliográfica: 1. Contreras et al. 2001, 2. Contreras et al. 2003, 3. Yáñez 2006, 4. Mejenes et al. 2007, 5. Osborne et al. 2006, 6. Ejemplares de herbarios (MEXU, IEB, FCME, HGOM y ENCB), 7. Recolectas de campo realizadas por Aurelia Vite Reyes, María T. Pulido y Juan C. Flores-Vázquez. *cultivado. Índice de diversidad expresado en número de especies/ln área. 
recolectado por M. González Ledesma). Los nuevos registros municipales son: Z. fischeri en Atlapexco, Chapulhuacán, Tepehuacán de Guerrero y Tlanchinol; Z. loddigesii en Chapulhuacán, Huautla, Huazalingo y Xochiatipan; Dioon edule en Chapulhuacán, Pisaflores, La Misión y Pacula; C. latifolia en Chapulhuacán; C. fuscoviridis en Chapulhuacán, La Misión, Juárez Hidalgo, Tlahuiltepa, Tepehuacán de Guerrero, Tianguistengo, Xochicoatlán y San Bartolo Tutotepec (Cuadro 1).

Las especies distribuidas en un mayor número de municipios son: $C$. mexicana en diez, C. fuscoviridis en diez, Z. loddigesii en nueve, $D$. edule en seis, $Z$. fischeri en cinco, mientras que las especies restantes están distribuidas en uno o dos municipios (Cuadro 1). Los datos de campo muestran que los tipos de vegetación que albergan mayor número de especies son la selva mediana $(C$. latifolia, $C$. fuscoviridis y $Z$. loddigesii) y el encinar $(C$. fuscoviridis, C. sabatoi y D. edule), mientras que solo hay una especie en la selva alta $(Z$. fischeri), la selva baja (D. edule), el bosque de encino pino (C. sabatoi) y el bosque mesófilo de montaña (C. fuscoviridis).

Comparación de la riqueza de Zamiáceas en Hidalgo y en otras áreas: Con este trabajo se evidencia que Hidalgo se posiciona como la cuarta entidad federativa con mayor riqueza de especies en México, después de Veracruz, Chiapas y Oaxaca. Además, su riqueza es mayor que la de países centroamericanos como Honduras, Belice y Costa Rica (Cuadro 2).

Planteamiento de la estrategia estatal de conservación: Las zonas prioritarias a conservar son las siguientes (Cuadro 3):

a. Municipios con riqueza sobresaliente de cícadas: Por la alta riqueza de Chapulhucán y Pisaflores debería crearse allí un área núcleo del CBBMM (Cuadro 3, Zona \#1), que incluya una localidad de Chapuluhacán donde coexisten simpátricamente cuatro especies, un caso excepcional para México. Esta zona núcleo permitiría incrementar la conectividad ecosistémica con la Reserva de la Biósfera Sierra Gorda (Querétaro).

b. Localidades con riqueza sobresaliente de cícadas: En una comunidad de Pisaflores se observó una población destacable de $D$. edule, con individuos de más de un metro de altura, que son ramoneados por el ganado, por lo que se propone la conservación de esa zona (Cuadro 3, Zona \#2).

CUADRO 2

Superficie, número de especies de cícadas (por género y total) e índice de diversidad (IB) para diferentes estados de la República Mexicana y otros países (*)

TABLE 2

Area, cycad species richness (species number per genus and total) and diversity index (IB) for several Mexican states and other countries $(*)$

\begin{tabular}{|c|c|c|c|c|c|}
\hline Entidad o país & $\begin{array}{l}\text { Superficie } \\
\left(\mathrm{km}^{2}\right)\end{array}$ & $\begin{array}{c}\text { Ceratozamia, Dioon, } \\
\text { Zamia (\# especies) }\end{array}$ & $\begin{array}{l}\text { Número de } \\
\text { especies }\end{array}$ & IB & Fuente bibliográfica \\
\hline Veracruz & 78815 & $9,2,7$ & 18 & 1.60 & Vovides et al. 1983, Hill et al. 2007 \\
\hline Chiapas & 73289 & $9,1,7$ & 17 & 1.52 & Hill et al. 2007 \\
\hline Oaxaca & 93793 & $6,7,4$ & 17 & 1.48 & Hill et al. 2007 \\
\hline Hidalgo & 20905 & $4,1,3$ & 8 & 0.80 & Contreras et al. 2003, Osborne et al. 2006, Este trabajo \\
\hline Querétaro & 11978 & $3,1,2$ & 6 & 0.64 & Vovides 1999 \\
\hline Honduras * & 112090 & $1,1,4$ & 6 & 0.52 & Nicolalde et al. 2009, Haynes et al. 2007 \\
\hline Belice * & 22965 & $1,0,4$ & 5 & 0.50 & Hill et al. 2007, Calonje 2009, Calonje et al. 2009 \\
\hline Costa Rica * & 51100 & $0,0,5$ & 5 & 0.46 & Rafael Acuña Castillo com. pers., Hill et al. 2007 \\
\hline San Luís Potosí & 60983 & $3,1,1$ & 5 & 0.45 & Hill et al. 2007 \\
\hline
\end{tabular}


CUADRO 3

Descripción de las zonas propuestas en la estrategia estatal de conservación de cícadas para el estado de Hidalgo

TABLE 3

Description of the zones proposed in the Cycad State Strategy in the State of Hidalgo

\begin{tabular}{|c|c|c|c|c|c|c|}
\hline $\begin{array}{l}\text { Zona } \\
\text { número }\end{array}$ & Municipio & Vegetación & $\begin{array}{l}\text { Mecanismo de } \\
\text { conservación }\end{array}$ & $\begin{array}{l}\text { Área } \\
\text { (ha) }\end{array}$ & $\begin{array}{l}\text { Especies } \\
\text { a conservar }\end{array}$ & Justificación \\
\hline 1 & $\begin{array}{l}\text { Chapulhuacán, } \\
\text { Pisaflores }\end{array}$ & $\begin{array}{l}\text { Selvas } \\
\text { medianas }\end{array}$ & ANP (nueva) & 10000 & $\begin{array}{l}\text { C. latifolia, C. fuscoviridis, } \mathrm{I} \\
\text { C. mexicana, D. edule, } \\
\text { Z. fischeri, } \\
\text { Z. loddigesii }\end{array}$ & $\begin{array}{l}\text { Presencia de seis de las ocho } \\
\text { especies reportadas para Hidalgo }\end{array}$ \\
\hline 2 & Pisaflores & Encinar & $\begin{array}{l}\text { UMA o reserva } \\
\text { comunitaria }\end{array}$ & 5 & D. edule & $\begin{array}{l}\text { Altas densidades de adultos muy } \\
\text { viejos }\end{array}$ \\
\hline 3 & Zimapán & Encinar & ANP (existente) & 100 & C. sabatoi & $\begin{array}{l}\text { Únicos sitios donde se ha } \\
\text { encontrado a la especie endémica } \\
\text { de Hidalgo y Querétaro }\end{array}$ \\
\hline 4 & Molango & Mesófilo & $\begin{array}{l}\text { UMA o reserva } \\
\text { comunitaria }\end{array}$ & 30 & C. fuscoviridis & $\begin{array}{l}\text { Alto empleo de las hojas para } \\
\text { múltiples usos }\end{array}$ \\
\hline 5 & Tlanchinol & $\begin{array}{l}\text { Mesófilo y } \\
\text { selva alta }\end{array}$ & $\begin{array}{l}\text { UMA o reserva } \\
\text { comunitaria }\end{array}$ & 30 & C. fuscoviridis & $\begin{array}{l}\text { Alto empleo de las hojas para } \\
\text { múltiples usos }\end{array}$ \\
\hline 6 & Huejutla & Zona agrícola & $\begin{array}{l}\text { UMA o reserva } \\
\text { comunitaria }\end{array}$ & 30 & Z. vazquezii & $\begin{array}{l}\text { Único sitio donde se ha } \\
\text { encontrado a la especie en la } \\
\text { entidad }\end{array}$ \\
\hline 7 & Chapulhuacán & Selva mediana & $\begin{array}{l}\text { UMA o reserva } \\
\text { comunitaria }\end{array}$ & 30 & C. latifolia & $\begin{array}{l}\text { Es el único sitio donde se ha } \\
\text { reportado la especie en el estado }\end{array}$ \\
\hline 8 & $\begin{array}{l}\text { San Bartolo } \\
\text { Tutotepec o } \\
\text { Huehuetla }\end{array}$ & Mesófilo & $\begin{array}{l}\text { Reserva } \\
\text { comunitaria }\end{array}$ & 100 & C. fuscovoridis & $\begin{array}{l}\text { Está en el límite de la } \\
\text { distribución sur de la especie, } \\
\text { podría ser importante } \\
\text { genéticamente }\end{array}$ \\
\hline 9 & A definir & & & 1000 & & Nuevas zonas a definir \\
\hline TOTAL & & & & 11325 & & \\
\hline
\end{tabular}

c. Respuesta de las especies ante el cambio de uso de suelo: C. fuscoviridis, Z. fischeri, $Z$. loddigesii y $D$. edule fueron observadas en pequeños parches de vegetación natural rodeados por pastizales o campos agrícolas, de hecho, algunas poblaciones de $D$. edule se encontraron en basureros, lo que sugiere que soportan mayores umbrales de disturbio antrópico, aunque debe estudiarse a fondo el efecto de este sobre sus poblaciones. Por el contrario, C. sabatoi solo fue encontrada en zonas conservadas (dentro del ANP llamada Parque Nacional los Mármoles) y muy rara vez en pequeños parches de vegetación, lo cual hace pensar que es una especie más sensible al disturbio antrópico.
Por lo anterior, C. sabatoi debe conservarse preferentemente dentro de ANP (Cuadro 3, Zona propuesta \#3), mientras que C. fuscoviridis, Z. fischeri, Z. loddigesii y $D$. edule podrían ser conservadas en paisajes fragmentados, que también incluyan algunos relictos de bosque continuo para permitir el intercambio genético y el mantenimiento de las poblaciones a largo plazo.

d. Grado de presión humana sobre el recurso: Se sugiere crear UMAS o reservas comunitarias en las localidades hidalguenses donde las cícadas se usan más intensamente como productos no maderables (Cuadro 3, Zona propuesta \#4 y \#5). En Hidalgo la especie más usada actualmente 
es C. fuscoviridis de la cual se emplean las frondas para elaborar arcos decorativos, al igual que ocurre con $D$. edule. Otros usos incluyen el estróbilo de $Z$. fischeri como juguete y sus semillas como veneno (Vite 2010, Vite, Pulido \& Flores-Vázquez 2010).

e. Especies con distribución geográfica más restringida: A escala estatal, aquellas de distribución más reducida son C. fuscoviridis, que habita solo en Hidalgo y $C$. sabatoi en Hidalgo y Querétaro. A escala municipal, las especies distribuidas en menos municipios son C. latifolia, Z. vazquezii y C. sabatoi (Cuadro 1). Por tanto, se propone la zona \#6 y \#7 (Cuadro 3) para la conservación de $Z$. vazquezii y C. latifolia. Además, se plantea la zona \#8 (Cuadro 3) para C. fuscoviridis, teniendo en cuenta que las poblaciones que están en el límite de su distribución pueden tener particularidades evolutivas importantes.

Adicionalmente, esta estrategia de conservación deberá concertarse tanto con los pobladores locales, como con los organismos gubernamentales estatales y federales encargados de la conservación de la biodiversidad. Es posible que durante ese proceso surjan nuevas áreas propuestas por estos sectores, por lo cual se consideró un área adicional de al menos 1000 ha (Cuadro 3, Zona \#9). De esta forma, el área propuesta de conservación incluye en total nueve zonas, que cubren aproximadamente 11325ha.

\section{DISCUSIÓN}

Estrategia estatal de conservación: Se plantea conservar nueve zonas focales, elegidas con base en los elementos descritos, localizadas en siete municipios que incluyen: encinares, bosque mesófilo, zonas agrícolas y selvas altas y medianas. Las nueve zonas propuestas suman un área de 11 325ha, que contribuiría a la conservación de las Zamiáceas presentes en la zona meridional de la SMO. Dado que es un área relativamente pequeña y que está espacialmente separada, se piensa que es una aproximación realista de implementar. No se proporciona un mapa detallado de las zonas a conservar para evitar su posible saqueo. Debe resaltarse que la importancia biológica de la Zona \#1 fue también notada por Luna et al. (2006) para otras especies amenazadas.

Para lograr esta propuesta de conservación fue indispensable realizar un estudio a escala municipal, con amplios recorridos de campo. Por tanto, aunque los estudios a escala nacional son imprescindibles (por ejemplo, permiten obtener patrones generales y regiones biogeográficas de mayor riqueza), deben complementarse con estudios a escalas locales (March et al. 2009). De esta forma, las decisiones de conservación deben realizarse de modo multiescalar, pasando de tendencias generales a investigaciones detalladas en sitios sobresalientes.

Uno de los criterios importantes tomados en esta estrategia fue tener en cuenta los usos que hacen los pobladores locales de estas plantas. Por ello, en esas zonas se sugiere crear UMAS o reservas comunitarias buscando su aprovechamiento sostenible. Se debería realizar experimentos de defoliación para optimizar el uso de sus frondas.

Además, es importante reconocer y considerar en el diseño de la estrategia el nivel de amenaza de las especies particulares. Si se toma en cuenta esto, se reporta a $C$. fuscoviridis y Z. vazquezii en peligro crítico (CR), C. sabatoi y $Z$. fischeri en peligro (EN), C. latifolia y C. mexicana como vulnerables (VU), D. edule y Z. loddigesii casi amenazadas (NT) (Stevenson, Vovides \& Chemnick 2003).

Debe enfatizarse que la estrategia de conservación planteada es novedosa metodológicamente puesto que: 1) combina simultáneamente diversas figuras de conservación, tales como zonas de conservación comunitarias, ANP, UMA; 2) plantea conservar múltiples polígonos de diversos tamaños y discontinuos especialmente; 3) pretende tomar en cuenta las particularidades específicas de las especies (umbral de perturbación, abundancia, distribución geográfica, etc); 4) incorpora el uso 
antrópico de las especies de interés. Lo anterior se contrapone a las estrategias que asumen la conservación solo mediante ANP, que plantean polígonos de conservación siempre grandes y continuos, y que generalmente no incorpora el papel dual del ser humano en el manejo y mantenimiento de la biodiversdad. El método aquí presentado se recomienda para proponer estrategias de conservación en este y otros grupos biológicos de interés. Aun así, solo es posible hacerlo en los casos que se disponga de suficiente información sobre la distribución local de las especies, lo cual implica un notorio esfuerzo de muestreo en campo.

Elementos a profundizar: El cambio de uso del suelo es una de las mayores amenazas para las cícadas (INE-SEMARNAP 2000), por lo que falta incorporar a la presente propuesta un análisis detallado de las tendencias de cambio de las coberturas y de uso del suelo, lo cual es básico para planear el ordenamiento ecológico de un territorio (Azuela et al. 2008) y para la conservación de su biodiversidad. Para Hidalgo no se dispone de esta información, aunque en los recorridos de campo fue evidente que la parte norte del estado está siendo alterado a tasas relativamente altas, debido principalmente a la expansión ganadera y a la apertura de caminos. En este sentido, el índice de riesgo de deforestación generado por el INECC (2012) sugiere que este es relativamente bajo en la mayor parte de la región hidalguense de la SMO, aunque es particularmente alto en el área de Chapulhuacán y Pisaflores. Esto sugiere que es urgente realizar esfuerzos de conservación en esos dos municipios.

Adicionalmente, sería útil disponer de estudios detallados sobre el estado de las poblaciones de cícadas, el efecto de la fragmentación y el cambio de uso de suelo sobre estas. En este sentido, se sabe que en pequeños fragmentos existen densidades de 0.358individuos/ $\mathrm{m}^{2}$ de $C$. fuscoviridis en Chapulhuacán y hasta 0.945 individuos $/ \mathrm{m}^{2}$ en San Bartolo Tutotepec, que presenta alta abundancia de plántulas, aunque en algunos sitios no se observan plantas de algunas tallas mayores (Vargas 2011). Es relevante hacer estudios de la demografía de las especies, para entender si las poblaciones presentes tienen el potencial de mantenerse a largo plazo.

Debe estudiarse más a fondo a C. fuscoviridis pues es casi desconocida científicamente. Se requiere analizar detalladamente las poblaciones de esta especie, considerada endémica de Hidalgo (aunque tal vez se distribuya en estados vecinos), su comportamiento demográfico y su variación genética, pues es posible que se estén observando numerosos relictos de una distribución continua del pasado. Nótese que la especie estaba registrada solo en la zona de "Tlanchinol-Molango" (Osborne, Stevenson \& Vovides 2006), mientras que el presente trabajo comprueba que habita en diez municipios, lo que refleja que se requiere de más estudios sobre ésta.

Por lo anterior, se hacen recomendaciones que pueden ser aplicadas por los manejadores de la ANP propuesta denominada CBBMM. En primer lugar, teniendo en cuenta la gran fragmentación de la zona y la distribución de la biodiversidad en ese mosaico, se sugiere combinar simultáneamente varias estrategias de conservación. Por un lado podrían manejarse uno o más tipos de ANP, según convenga a nivel práctico. Por otro lado, como el PNC sugiere, es importante incorporar zonas con Zamiáceas bajo el esquema de UMA. Este instrumento de conservación se incluyó en la presente propuesta.

Debe incorporarse un estudio y entendimiento del contexto de política pública que puede afectar la conservación de la biodiversidad del territorio en el largo plazo. Por ejemplo, el Parque Nacional Los Mármoles está en proceso de recategorización, motivo por el cual se está elaborando un plan de manejo en el que la zonificación está dando prioridad a los requerimientos de zonas de aprovechamiento minero (de mármol), aún por encima de lo decretado para su conservación biológica. Por lo tanto, la dimensión política evidentemente es uno de los elementos fundamentales para lograr estrategias reales de conservación. 
Adicionalmente, deben promoverse estrategias de conservación basadas en las comunidades, lo cual puede ser muy eficiente especialmente en contextos de mantenimiento de las estructuras de organización comunitaria de los recursos y del territorio (Camacho, Martin \& Del Campo-García 2010); también deben organizarse talleres y pláticas para los pobladores locales acerca de la importancia evolutiva y cultural de estas plantas.

Se sugiere que el PNC priorice las zonas del país con mayor valor para la conservación de cícadas y que proporcione algunas recomendaciones generales. De esta forma, en las zonas de mayor interés nacional podrían replicarse estrategias semejantes a la aquí desarrollada. Es importante señalar que la implementación de estas estrategias debe hacerse de manera conjunta entre las comunidades locales, la academia y los organismos estatales y federales encargados de la conservación de la biodiversidad.

\section{AGRADECIMIENTOS}

Agradecemos el apoyo del proyecto FOMIX-HGO-2008-95828 "Diversidad biológica del Estado de Hidalgo (segunda fase)" por el financiamiento otorgado para el trabajo de campo y el apoyo económico dado al primer autor (AVR). Al Herbario Nacional de México por permitir la consulta de la colección de cícadas. A Andrew Vovides por su invaluable ayuda en la determinación del material botánico y asesoría. A los habitantes de las comunidades por su ayuda y explicaciones en campo. Por compartir sus observaciones de campo a Sol Mejenes, Manuel González-Ledesma, Claudia Hornung y Juan Márquez. Por su ayuda en campo a Maricela Vargas y Gilberto Mendoza. Por sus comentarios al manuscrito a Norberto Martínez y Numa Pavón. Por la corrección de estilo a Consuelo Cuevas Cardona. Por la traducción al inglés a Irene Sánchez-Gallen. A los revisores anónimos por enriquecer notablemente este manuscrito.

\section{RESUMEN}

México es el segundo país con mayor diversidad de cícadas en el mundo. En él, la Sierra Madre Oriental (SMO) es una de las regiones biogeográficas con mayor riqueza de estas plantas. El objetivo de este estudio fue plantear la estrategia de conservación de cícadas para el estado de Hidalgo, ubicado en la parte sur de la SMO; para esto se revisó la literatura científica, se consultaron los principales herbarios de México y se hicieron recorridos de campo. Los resultados muestran que Hidalgo alberga tres géneros y ocho especies: Ceratozamia fuscoviridis, C. latifolia, C. mexicana, C. sabatoi, Dioon edule, Zamia fischeri, Z. loddigesii, Z. vazquezii, posicionándose en el cuarto lugar nacional en riqueza de cícadas. Las especies se distribuyen en 26 municipios. Se encontró que la diversidad de cícadas es mayor a la de otras entidades con mayor superficie e incluso a la de algunos países de Centroamérica. La estrategia estatal de conservación de cícadas aquí desarrollada propone conservar cerca de 11 325 ha, distribuidas en siete municipios, bajo un esquema mixto de conservación. Esta propuesta será útil para que las instancias gubernamentales establezcan nuevas ANP's en la región biogeográfica de la SMO.

Palabras clave: cícadas, conservación local, Programa Nacional de Cícadas, Sierra Madre Oriental, UMAS.

\section{REFERENCIAS}

Azuela, A., M.A. Cancino, C. Contreras \& A. Rabasa. 2008. Una década de transformaciones en el régimen jurídico del uso de la biodiversidad, p. 259-282. In J. Sarukhán, P. Koleff, J. Carabias, J. Soberón, R. Dirzo, J. Llorente, G. Halffter, R. González, I. March, A. Mohar, S. Anta \& J. De la Masa. (Eds.). Capital natural de México, Vol. III: Políticas públicas y perspectivas de sustentabilidad. CONABIO, México.

Calonje, M. 2009. A new cliff-dwelling species of Zamia (Zamiaceae) from Belize. J. Bot. Res. Inst. Texas: 23-29.

Calonje, M., J. Meerman, P. Griffith \& G. Hoese. 2009. A new species of Zamia (Zamiaceae) from the Maya mountains of Belize. J. Bot. Res. Inst. Texas 3: 31-41.

Camacho, C., G. Martin \& C. Del Campo-García. 2010. Fortalecimiento de áreas de conservación comunitarias e indígenas a través de la Etnobiología, p. 30-35. In A. Moreno, M.T. Pulido, R. Mariaca, R. Valadéz Azúa, P. Mejía Correa \& T.V. Gutiérrez Santillán (Eds.). Sistemas biocognitivos tradicionales: paradigmas en la conservación biológica y el fortalecimiento cultural. México: Universidad Autónoma del Estado de Hidalgo, Asociación Etnobiológica Mexicana y Sociedad Latinoamericana de Etnobiología. 
Canseco-Márquez, L., F. Mendoza-Quijano \& M.G. Gutiérrez-Mayén. 2004. Análisis de la distribución de la herpetofauna, p. 417-437. In I. Luna, J.J. Morrone \& D. Espinosa (Eds.). Biodiversidad de la Sierra Madre Oriental. México, Ciudad de México, Las Prensas de Ciencias, UNAM-CONABIO.

Congreso de la Unión. 1988. Ley General del Equilibrio Ecológico y la Protección al Ambiente. Diario Oficial de la Federación, 28 de enero de 1988, últimas reformas 4 de junio de 2012.

Contreras, R., I. Luna \& O. Alcántara. 2001. Registro de Zamia fischeri (Zamiaceae) en Hidalgo, México. Anales Inst. Biol. UNAM, Ser. Bot. 72: 115-118.

Contreras, R., I. Luna \& O. Alcántara. 2003. Zamiaceae en Hidalgo, México. Anales del Instituto de Biología. Universidad Nacional Autónoma de México, Ser. Bot. 74: 289-301.

Haynes, J., L. Whithelock, B. Shutzman \& R. Adams. 2007. A new species of Ceratozamia from Honduras (Cycadales: Zamiaceae). Cycad Newsletter 31: $16-21$.

Hill, K.D., D. Stevenson \& R. Osborne. 2007. The world list of cycads. Proceedings of the 7th International Conference on Cycad Biology (CYCAD 2005), Xalapa, Mexico. January 2005. Memoirs New York Bot. Gard. 97: 454-483.

Instituto Nacional de Ecología y Secretaría de Medio Ambiente y Recursos Naturales (INE-SEMARNAP) 2000. Proyecto para la protección, conservación y recuperación de la familia Zamiaceae (Cycadales) de México. INE-SEMARNAP. México D.F. (Consultado: 5 febrero 2013, http://www2.ine.gob.mx/publicaciones/consultaPublicacion.html?id_pub=183 ]).

Instituto Nacional de Ecología y Cambio Climático (INECC). 2012. Índice de presión Económica (Riesgo) de deforestación (Consultado: 5 febrero 2013, http://www.ine.gob.mx/irdef).

Instituto Nacional de Estadística Geografía e Informática (INEGI). 1992. Síntesis Geográfica del Estado de Hidalgo. INEGI, México.

Juárez, H.S.A.C., R. Contreras \& I. Luna. 2009. Biogeographic analysis of endemic cacti of the Sierra Madre Oriental, Mexico. Biol. J. Linn. Soc. 97 373-389.

Luna, I., O. Alcántara, J.J. Morrone \& D. Espinosa. 2000. Track analysis and conservation priorities in the cloud forests of Hidalgo, Mexico. Diversity Distrib. 6: $137-143$

Luna, I. \& O. Alcántara. 2004. Florística del bosque mesófilo de montaña de Hidalgo, p. 169-191. In I. Luna, J.J. Morrone \& D. Espinosa. (Eds.). Biodiversidad de la Sierra Madre Oriental. Ciudad de México, México. Las Prensas de Ciencias, UNAM-CONABIO.
Luna, I., J.J. Morrone \& D. Espinosa. 2004. Biodiversidad de la Sierra Madre Oriental. Ciudad de México, México. Las Prensas de Ciencias, UNAM-CONABIO.

Luna, I., O. Alcántara, R. Contreras \& A. Ponce. 2006. Biogeography, current knowledge and conservation of threatened vascular plants characteristic of Mexican temperate forests. Biodiversity Conservation 15: 3773-3799.

Márquez, J. \& J.J. Morrone. 2004. Relaciones biogeográficas basadas en la distribución de Coleoptera (Insecta), p. 375-392. In I. Luna, J.J. Morrone \& D. Espinosa. (Eds.). Biodiversidad de la Sierra Madre Oriental. Ciudad de México, México. Las Prensas de Ciencias, UNAM-CONABIO.

March, I.J., M.A. Carvajal, R.M. Vidal, S.E. San Román \& G. Ruiz. 2009. Planificación y desarrollo de estrategias para la conservación de la biodiversidad. In J. Sarukhán, P. Koleff, J. Carabias, J. Soberón, R. Dirzo, J. Llorente, G. Halffter, R. González, I. March, A. Mohar, S. Anta \& J. De la Masa (Eds.). Capital Natural de México, Vol. II: Estado de conservación y tendencias de cambio (545-573). CONABIO. México.

Mas, J.F. \& B.A. Pérez. 2005. La representatividad del Sistema Nacional de Áreas Protegidas (SINAP). Gaceta Ecológica, 74, 5-14. (Consultado: 5 febrero 2013, http://ww2.ine.gob.mx/publicaciones/gacetas/460/ sinap.html).

Mejenes, S.M., T.J. Barragán \& B.M. Hernández. 2007. Cícadas de la Región Sierra y Huasteca de Hidalgo. XVII Congreso Mexicano de Botánica. Zacatecas, México.

Nicolalde, F., A.P. Vovides \& D.W. Stevenson. 2009. Taxonomic revision of Zamia in Mega-Mexico. Brittonia 61: 301-335.

Osborne, R., D.W. Stevenson \& A.P. Vovides. 2006. What is Ceratozamia fuscoviridis? Delpinoa 48: 5-10.

Rzedowski, J. 2006. Vegetación de México. México: Comisión Nacional para el Conocimiento y Uso de la Biodiversidad. (Consultado: 5 febrero 2013, http:// www.biodiversidad.gob.mx/publicaciones/librosDig/ pdf/VegetacionMx_Cont.pdf).

Sarukhán, J., P. Koleff, J. Carabias, J. Soberón, R. Dirzo, J. Llorente, G. Halffter, R. González, I. March, A. Mohar, S. Anta \& J. De la Masa. 2009. Capital Natural de México. Síntesis: conocimiento actual, evaluación y perspectivas de sustentabilidad. CONABIO, México.

SEMARNAT. 2010. Norma Oficial Mexicana NOM059-SEMARNAT-2010, Protección ambiental-Especies nativas de México de flora y fauna silvestres-Categorías de riesgo y especificaciones para su inclusión, exclusión o cambio-Lista de especies en riesgo. Diario Oficial de la Federación, 30 de diciembre. 
Squeo, F., L. Cavieres, G. Arancio, J. Novoa, O. Matthei, C. Marticorena, R. Rodríguez, M. Arroyo \& M. Muñoz. 1998. Biodiversidad de la Flora Vascular en la Región de Antofagasta, Chile. Rev. Chil. Hist. Nat. 71: 571-591.

Stevenson, D.W., A.P. Vovides \& J. Chemnick. 2003. Regional Overview: New World, p. 31-38. In J. Donaldson (Ed.). Cycads. Status Survey and Conservation Action Plan. Switzerland and Cambridge, UK: IUCN/SSC Cycad Specialist Group.

The Cycad Pages. 2009. Royal Botanical Garden Sydney. (Consultado, 5 febrero 2013, http://plantnet.rbgsyd. nsw.gov.au/PlantNet/cycad/).

Vargas, M. 2011. Demografía y variación morfológica foliar de Ceratozamia fuscoviridis Moore (Zamiaceae) en el estado de Hidalgo. Tesis de Licenciatura, Universidad Autónoma del Estado de Hidalgo, México.

Vite, A. 2010. Etnobotánica de cícadas en Hidalgo y algunos aspectos demográficos de Ceratozamia fuscoviridis D. Moore. Tesis de Licenciatura, Universidad Autónoma del Estado de Hidalgo, México.
Vite, A., M.T. Pulido \& J.C. Flores-Vázquez. 2010. Aspectos etnobotánicos de las cícadas en algunas zonas de Hidalgo, México, p. 481-486. In A. Moreno, M.T. Pulido, R. Mariaca, R. Valadéz Azúa, P. Mejía Correa \& T.V. Gutiérrez Santillán (Eds.). Sistemas biocognitivos tradicionales: paradigmas en la conservación biológica y el fortalecimiento cultural. México: Asociación Etnobiológica Mexicana, Global Diversity foundation, Universidad Autónoma del Estado de Hidalgo, Colegio de la Frontera Sur y Sociedad Latinoamericana de Etnobiología.

Vovides, A.P., J.D. Rees \& M. Vázquez. 1983. Zamiaceae. Flora Veracruz 26: 4-11.

Vovides, A.P. 1999. Flora del Bajío y de Regiones Adyacentes (Zamiaceae). Instituto de Ecología A.C., 71, 1-17.

Vovides, A.P. 2000. México: segundo lugar mundial en diversidad mundial de cícadas. Biodiversitas 31: 1-9.

Yáñez, L. 2006. Las cícadas, biología y conservación en México. Universidad Autónoma de Chapingo, Estado de México, México. 
